\title{
A pesquisa sobre segurança alimentar e nutricional no Brasil de 2000 a 2005: tendências e desafios
}

\author{
Research on food and nutritional security \\ in Brazil from 2000 to 2005: trends and challenges
}

Shirley Donizete Prado ${ }^{1}$

Silvia Ângela Gugelmin ${ }^{1}$

Rubem Araújo de M attos ${ }^{2}$

Juliana Klotz Silva ${ }^{1}$

Priscila dos Santos Gomes Olivares ${ }^{1}$

\footnotetext{
${ }^{1}$ Departamento deN utrição Social, Instituto de Nutrição, Universidadedo Estado do Rio deJaneiro. RuaSão Francisco Xavier 524, Pavilhão João Lyra Filho, 12은 andar, Bloco D, sala 12028, M aracanã. 20559-900 Rio deJaneiro RJ. shirley_prado@terra.com.br ${ }^{2}$ Departamento de Planejamento e Administração em Saúde, Instituto deM edicina Social, Universidade do Estado do Rio deJaneiro.
}

Abstract Discussion of Research on Food and Nutritional Security (FNS) in Brazil started with the Directory of Brazilian Research Groups from the National Council for Scientific and Technological Development (CNPq). Searches using the expression "food security" allowed the identification of 72 groups in 2005. Proportions between researchers and students suggest little dynamism and consolidation of such research. Groups in the area of Food Science and Technology, and those concerned with food quality are predominant; then there is the Nutrition area, mainly discussing the nutritional state of population groups; in thefield of Humanities there is a small number of groups discussing political, economic and social aspects within FNS'snational and regional scopes. It seems that investments in the integration between food and nutrition components of research on FNS are necessary, in order to attenuatedifferen ces between the economic (food security) and the social focus (human rights to a healthy nutrition).

Key words Food and nutritional security, Research groups, Research lines, Food and nutrition policies, Trends of Brazilian research
Resumo A pesquisa sobre segurança alimentar e nutricional (SAN) no Brasil é discutida a partir do Diretório dos Grupos de Pesqui sa no Brasil do CN Pq. Buscas utilizando a expressão "segurança alimentar" possibilitaram a identificação de 72 grupos em 2005. As proporções entre pesquisadores e estudantes sugerem baixos dinami smo econsolidação dessa pesquisa. Predominam grupos na área de ciência e tecnologia dos alimentos e mais voltados para a qualidade do alimento; segue-sea área da Nutrição discutindo, principalmente, 0 estado nutricional de grupos populacionais; na área das humanidades, há um pequeno número de grupos que discute aspectos políticos, econômicos e sociais em âmbitos nacional e regional da SAN. Parecem ser necessários investimentos na integração entre os componentes alimentar e nutricional da pesquisa sobre SAN, buscando reduzir o diferencial entre os focos econômico (segurança do alimento) e social (direito humano à alimentação saudável).

Palavras-chave Segurança alimentar e nutricional, Grupos de pesquisa, Linhas de pesquisa, Políticas de alimentação e nutrição, Tendências da pesquisa brasileira 
Introdução

Esteestudo faz parte deum projeto maior intitulado "A pesquisa sobreAlimentos, Alimentação e Nutrição no Brasil|" ${ }^{1}$ cuja primeira etapa tem por objeto a pesquisa sobre Segurança Alimentar e Nutricional (SAN).

Segundo Valente², SAN consiste em garantir a todos condições de acesso a alimentos básicos seguros e de qualidade, em quantidade suficiente, de modo permanente e sem comprometer 0 acesso a outras necessidades essenciais, com base em práticas alimentares saudáveis, contribuindo assim para uma existência digna em um contexto de desenvolvimento integral da pessoa humana.

A implementação da SAN implica ações articuladas envolvendo vários setores: política econômica, emprego e renda, políticas de produção agroalimentar (políticas agrária, de produção agrícola e agroindustrial), comercialização, distribuição, acesso e consumo de alimentos, com perspectivas de descentralização e diferenciação regional; ações emergenciais contra a fome; ações de controle da qualidade dos alimentos; diagnóstico e monitoramento do estado nutricional e desaúde de populações; estímulo a práticas al imentares saudáveis, além da valorização das culturas locais e regionais.

Como um objetivo nacional básico e estraté gico, a SAN deve permear e articular políticas e ações de todos os níveis de governo e ser perseguida por toda a sociedade, comprometen do diferentes segmentos sociais, seja em parceria com os distintos níveis de governo ou em iniciativas cidadãs ${ }^{3}$.

Ao tratar a questão alimentar como direito humano a ser atendido a partir de referenciais éticos e envolvendo questões estruturais, como 0 enfrentamento às desigualdades sociais e econômicas que marcam a sociedade brasileira, SAN corresponde, indiscutivelmente, a conceito de grande amplitude.

Os debates sobre SAN têm se tornado cada vez mais intensos e amplos nos últimos anos e são crescentes as demandas referentes à produção de conhecimentos e saberes que apresentem potencial de contribuição a esse campo de interesse. É o que se pode identificar em recomendações do Consel ho Nacional de SegurançaA Alimentar e Nutricional (CON SEA) presentes em textos oficiais que subsidiaram a II Conferência N acional de Segurança Alimentar e Nutricional ${ }^{4}$ como, por exemplo, a que sugere incluir "a te mática da alimentação como um dos direitos humanos nos diferentes espaços de formação edu- cacional e de pesquisa" - e também em seu relatório final 5 .

As agências nacionais de fomento à pesquisa, por sua vez, intensificaram, recentemente, chamadas para apresentação de projetos de pesquisa a serem financiadas den tro do escopo da SAN 6 . A constituição de uma rede de Conselhos Estaduais eM unicipais de SAN ea realização cada vez mais frequente de eventos científicos (seminários, simpósios, cursos) ou de cunho mais intervencionista voltados para a formulação e avaliação de políticas de SAN também correspondem a importantes focos de demandas por informações que venham a fornecer subsídios e a propiciar fundamentação sólida para sua consecução.

Deve-se registrar a aprovação, em setembro de 2006, da lei' "que institui o Sistema Nacional deSegurança A limentar e N utricional com vistas em assegurar o direito humano à alimentação", como componente de fortalecimento das ações nesse campo e de aumento de necessidades de informações sistemáticas e confiáveis, o que, ao menos em parte, pode e deve ser obtido via produção de novos conhecimentos científicos.

Este artigo tem por objetivo geral identificar os grupos de pesquisa que compõem a massa crítica capaz de contribuir para debates, formulação de políticas e implementação de ações e avaliação de procedimentos no campo da SAN no Brasil.

São seus objetivos específicos descrever e discutir a dimensão desse parquecientífico, sua distribuição geográfica, períodos de criação, tendências relativas ao dinamismo eà consolidação dessa pesquisa no Brasil, sua inserção desses grupos de pesquisa em áreas do conhecimento, produção científica e temas investigados.

\section{M etodologia}

Uma fonte privilegiada de informações sobre a pesquisa brasileira corresponde ao Diretório dos Grupos de Pesquisa no Brasil do Conselho Nacional de Desenvolvimento Científico e Tecnológico $(\mathrm{CN} \mathrm{Pq})^{8}$ que realizou, até hoje, seis censos (1993, 1995, 1997, 2000, 2002 e 2004). Os resultados desses três últimos inventários estão disponíveis na Internet, como também a consulta sobre a base corrente de dados, queéatualizada continuamente.

Trata-se de uma fonte de dados secundários que possibilita o mapeamento de campos do conhecimento em vários aspectos de sua dinâmica. Além disso, seu caráter censitário convida ao apro- 
fundamento do conhecimento por meio das inúmeras possi bilidades de estudos empíricos relativos a campos não cobertos pelos dados, como, por exemplo, o financiamento ou a avaliação qualitativa da produção científica e tecnológica.

N essa linha, alguns estudos já foram empreendidos no Brasil abordando, por exemplo, o campo da epidemiologia ${ }^{9}$ e da geriatria e gerontologia ${ }^{10,11}$, resultando em avaliações e reflexões acerca da constituição desses campos, bem como em apresentação formal daqueles grupos ou redes de pesquisadores que se destacam pela qualidade de sua produção científica atual ou potencial.

O critério para o reconhecimento dos grupos que compõem a massa crítica em epidemiologia foi o desenvolvimento de, pelo menos, uma linha de pesquisa nessa subárea da tabela das áre as do conhecimento do CNPq, conforme definido pelo pesquisador líder de cada grupo ${ }^{8}$. Para geriatria egerontologia, foi adotado procedimento diferenciado, dada a inexistência de área ou subárea com essa designação nessa tabela; procedeu-se, nesse caso, a busca por palavras-chave associadas ao tema em questão ${ }^{10,11}$.

$\mathrm{A}$ árvore do conhecimento, tal como apresentada hoje pelo CN Pq, não contempla, em qualquer nível, possibilidade de associação de qualquer área do conhecimento à SAN. Assim, ficou descartada a via metodológica de caminhar a partir das grandes áreas e suas subdivisões. TrabaIhou-se, portanto, realizando buscas no diretório a partir da expressão "segurança alimentar" nos seguintes campos: nome do grupo, nome da linha de pesquisa e palavras-chave da linha de pesquisa. A utilização de outras palavras-chave associadas à SAN, como fome ou soberania al imentar, confirmou os resultados encontrados.

Uma busca realizada em outubro de 2005 na base corrente do Diretório dos Grupos de Pesquisa a partir da mesma palavra-chave indicou a existência de 72 grupos que mencionaram essa temática. $\mathrm{Nem}$ todos os registros presentes nos censos aparecem na base corrente e esta é, caracteristicamente, flutuantee dinâmica, uma vez que as informações aí presentes podem ser alteradas a qualquer momento; esses fatores conduzem a utilização pontual dos dados referentes a 2005 e sua interpretação correspondeà indicação detendências a serem confirmadas nos censos vindouros. Foram encontrados 41 grupos no censo de 2004, 12 em 2002 e 5 em 2000.

Foram incluídos somente os pesquisadores que participam de linhas de pesquisa que contêm a expressão de busca no nome da linha de pesquisa ou nas palavras-chave da linha de pesquisa.

Os resultados são caracterizados por algumas limitações. De um lado, subestimados na medida em que há grupos que trabalham com SAN, mas que não registraram o termo de busca nos campos aqui utilizados. Por outro, superestimados uma vez que, em uma mesma linha de pesquisa, pode haver pesquisadores que não trabal ham com o tema em questão e não há recursos metodológicos no diretório que permitam identificá-los.

A identificação da produção científica, tecnológica e artística foi implementada através dos campos denominados referência bibliográfica, título da produção e palavra-chave da produção; também foi utilizada a palavra-chave"segurança alimentar". Os dados disponíveis no diretório acerca da produção científica dos grupos com linhas de pesquisa sobre SAN são provenientes do curriculum vitae Lattes (CV Lattes) de cada pesquisador.

Essa produção científica é superdimensionada no interior diretório, como muito bem esclarecem Guimarães et al. ${ }^{9}$, em seu estudo sobre a pesquisa nacional no campo epidemiológico: A possibilidade de múltipla contagem incide sobre todos os registros de produção existentes no Diretório, para todas as áreas do conhecimento. Ela é consequente a duas situações: co-autorias num mesmo grupo são regi stradas quantas vezes estejam presentes nos CV; e pesquisadores que participam de mais de um grupo têm sua produção contada também mais de uma vez.

N esteestudo, foram eliminadas, manual mente, todas as possibilidades de múltipla contagem, de modo que os dados apresentados correspondem ao número efetivo de produtos registrados pelos pesquisadores em seus CV Lattes.

Foram privilegiados al guns dos itens da produção bibliográfica (artigo nacional, artigo internacional, livro e capítulo de livro) e também as atividades de orientação concluídas na pósgraduação (tese de doutorado e dissertação de mestrado concluídas) por apresentarem maisfácil acesso ao seu conteúdo e por terem passado pelo crivo de bancas examinadoras ou por processos de avaliação por pares ou comitês editoriais.

Entende-se que ao mencionar SAN no título do grupo, de linhas de pesquisa ou de palavraschave que caracterizam essas linhas, o grupo de pesquisa declara intenção de se dedicar ao estudo dessa temática. 


\section{Resultados ediscussão}

Os resultados apresentados na Tabela 1 apresentam um panorama da pesquisa nacional eofe recem uma visão da ordem de grandeza referente aos grupos de pesquisa que mencionam SAN nos registros efetuados no diretório.

Esses resultados indicam um crescente interesse pela temática SAN nos anos recentes. No conjunto do diretório, foi encontrado um crescimento do número de grupos de pesquisa de 1,6 vezes no período de 2000 a 2004 (de 11.760 para $19.470)^{12}$, enquanto que os grupos voltados para SAN cresceram 8,2 vezes.

Os grupos que mencionam SAN reapresentavam apenas $0,21 \%$ do total degrupos que compunham o diretório, algo efetivamente muito reduzido em face das grandiosas dimensões da necessária intersetorial idade que demanda a implementação da SAN no Brasil. Em 2000, a pes- quisa em epidemiologia representava $1,4 \%$ eaquela relativa à temática do envelhecimento humano correspondia a $1,2 \%$ do total de grupos de pesquisa no Brasil. A partir dessa comparação, parece razoável afirmar queéreduzi da a massa crítica voltada para a produção de conhecimento no campo da SAN.

Distribuição geográfica

dos grupos de pesquisa

A Tabela 2 mostra que há predominância dos grupos com linhas de pesquisa que mencionam SAN na Região Sudeste - cerca da metade dos grupos $(55,5 \%)$ situam-se nesses estados em 2005. Este resultado é coerente com a distribuição do parque científico nacional que, historicamente, consolidou-se nos estados de maior expressão política eeconômica?. Valores semelhantes a este foram encontrados nos estudos acerca

Tabela 1. Número de instituições, grupos, pesquisadores e pesquisadores doutores no total do Diretório ${ }^{12}$ segundo ano do censo e que mencionam segurança alimentar e nutricional ${ }^{8}$ segundo ano do censo e na base corrente em outubro de 2005, Brasil.

\begin{tabular}{|c|c|c|c|c|c|c|c|}
\hline & \multicolumn{6}{|c|}{ Censo } & \multirow{3}{*}{$\begin{array}{c}\text { Base } \\
\text { corrente } \\
\text { outubro de } \\
2005 \text { SAN }\end{array}$} \\
\hline & \multicolumn{2}{|l|}{2000} & \multicolumn{2}{|c|}{2002} & \multicolumn{2}{|c|}{2004} & \\
\hline & Diretório & SAN & Diretório & SAN & Diretório & SAN & \\
\hline Instituições & 224 & 5 & 268 & 9 & 335 & 34 & 52 \\
\hline Grupos de pesquisa & 11.760 & 5 & 15.158 & 12 & 19.470 & 41 & 72 \\
\hline Linhas depesquisa & a & 25 & 12.977 & 84 & 14.459 & 248 & 360 \\
\hline Pesquisadores(P) & 48.781 & 47 & 56.891 & 183 & 77.649 & 441 & 717 \\
\hline Estudantes & 59.357 & 39 & 61.872 & 51 & 102.913 & 196 & 413 \\
\hline Pesquisadores doutores (D) & 27.662 & 27 & 34.349 & 147 & 47.973 & 300 & 496 \\
\hline (D)/(P) em \% & 57 & 54 & 60 & 80 & 62 & 68 & 69 \\
\hline
\end{tabular}

a. Informação não disponível no diretório.

Tabela 2. Distribuição dos grupos de pesquisa no total do Diretório ${ }^{12}$ por ano do censo e dos grupos de pesquisa que mencionam segurança alimentar e nutricional ${ }^{8}$ por ano do censo e na base corrente em outubro de 2005 segundo região geográfica, Brasil.

\begin{tabular}{|c|c|c|c|c|c|c|c|}
\hline \multirow[b]{3}{*}{ Região geográfica } & \multicolumn{6}{|c|}{ Anos do censo } & \multirow{3}{*}{$\begin{array}{c}\text { Base } \\
\text { corrente } \\
\text { outubro de } \\
2005 \text { SAN }\end{array}$} \\
\hline & \multicolumn{2}{|c|}{2000} & \multicolumn{2}{|c|}{2002} & \multicolumn{2}{|c|}{2004} & \\
\hline & Diretório & SAN & Diretório & SAN & Diretório & SAN & \\
\hline Sudeste & 6.733 & 3 & 7.855 & 8 & 10.221 & 18 & 40 \\
\hline Sul & 2.317 & 0 & 3.630 & 1 & 4.580 & 9 & 14 \\
\hline Nordeste & 1.720 & 2 & 2.274 & 3 & 2.760 & 7 & 9 \\
\hline Centro-oeste & 636 & 0 & 809 & 0 & 1.139 & 3 & 5 \\
\hline Norte & 354 & 0 & 590 & 0 & 770 & 4 & 4 \\
\hline Total & 11.760 & 5 & 15.158 & 12 & 19.470 & 41 & 72 \\
\hline
\end{tabular}


da geronto-geriatria (59,7\% em 2004), da epidemiologia (60,8\% em 2000) eno total do diretório (52\% em 2004).

São Paulo, M inas Gerais eRio de Janeiro concentram juntos $54 \%$ dos grupos com linhas que referem pesquisa sobre SAN no ano de 2005.

Período de criação dos grupos de pesquisa

O número de grupos de pesquisa e a produção de conhecimento tem aumentado nas últimas décadas no Brasil em decorrência de importante desenvolvimento acadêmico marcado por aumento na titulação dos docentes das universidades e ampliação do número de programas de pós-graduação concomitante à el evação de sua qualidade acadêmica ${ }^{13,14}$.

Além da dinâmica específica da organização institucional da produção de conhecimentos, é necessário considerar o contexto do surgimento e desenvolvimento dos conceitos e práticas associadas à SAN no plano internacional e no Brasil.

As origens do conceito de segurança alimentar situam-se na Europa do início do século XX dentro da perspectiva de que cada país deveria produzir sua própria alimentação, buscando assim reduzir ao máximo eventual vulnerabilidade em face de situações adversas que implicassem risco à soberania nacional. Por volta dos anos quarenta, documentos oriundos de recém-criados organismos internacionais financeiros ou voltados para o campo da agricultura começaram a mencionar a assistência alimentar - via excedentes de alimentos - como parte da segurança alimentar. Após longo período, em que prevaleceu a ideia de que a criação e a manutenção de estoques de alimentos seria suficientepara dar conta de problemas como a fome que atingia parcelas numerosas das populações, ampliou-se conceito desegurança alimentar para incluir componentes como a oferta estável e 0 acesso a alimentos de qualidade, articulados à redistribuição de renda e de bens materiais; posteriormente, foram incorporadas discussões sobre a necessidade imperiosa de equidade social, justiça erelações éticas entre as gerações e modos de vida sustentáveis. Atualmente, a questão da alimentação étratada como um componente do direito à vida digna, à autodeterminação e à plena satisfação das necessidades básicas do ser humano².

No Brasil, as bases do conceito de segurança alimentar remontam a meados do século XX, quando Josué de Castro procedeu as primeiras sistematizações de abrangência nacional acerca da fome no Brasil| 2,3, 15,16.
Durante o período ditatorial, o cenário político nacional tornou-se adverso ao desenvolvimento de estudos sobre temáticas deforte cunho social como a fome. Pesquisas dessa natureza foram retomadas em torno dos anos setenta, por exemplo, através do Estudo Nacional de Despesa Familiar ${ }^{17}$, cujos resultados estavam, ainda, cercados por certo controle por parte do governo. Datam desse período tentativas de incorporação de aspectos alimentares enutricionais ao planejamento econômico, conduzidas sob a direção do Instituto Nacional de Alimentação e Nutrição (INAN $)^{16}$.

A progressiva movimentação social no sentido da consolidação das liberdades civis e de buscas de alternativas para superação dos graves dilemas sociais do país ocorreu de forma mais ou menos concomitante à proposição e/ou implementação de vários planos e programas relativos à alimentação e nutrição. $N$ a década de oitenta, 0 Ministério da Agricultura começou a registrar, formalmente, iniciativas mencionando a segurança alimentar. Nos anos noventa, realizou-se a I Conferência Nacional de Segurança Alimentar e Nutricional. Vários estudos tratando do perfil nutricional da população brasileira foram implementados em torno e a partir dessa época como, por exemplo, a Pesquisa Nacional de Saúde e Nutrição ${ }^{18}$, as Pesquisas de Orçamentos Familiares $^{19}$, o M apa da Fome ${ }^{20}$, entre outros ${ }^{21,22}$.

Com os resultados das eleições para o poder executivo federal realizadas em 2002, 0 enfrentamento à miséria eà fome passou a tema de interesse central do primeiro escalão governamental. Iniciativas dessa dimensão política eram inéditas até então. O Programa Fome Zero - uma proposta de política de segurança alimentar para o Brasil - corresponde ao grande destaque, a uma marca de identidade dessa gestão. D esde então, os debates e ações no campo da SAN vêm passando por intenso incremento. Em 2003, foi reinstalado o CONSEA, vinculado à Presidência da República, com a participação de grande número de ministros e de significativo número de representantes da sociedade indicados por movimentos sociais. A II Conferência Nacional de SegurançaAlimentar eN utricional efetivou-seem 2004, ano em que se realizou a primeira pesquisa nacional sobre insegurança alimentar - o M ódulo sobre Segurança Alimentar da Pesquisa Nacional por Amostra de Domicílios. Intensa mobilização marca a III edição da Conferência Nacional de Segurança Alimentar eN utricional em 2007.

Os eventos registrados acima, ainda que de forma pontual, indicam que SAN corresponde à temática candente e marcada por força instituci- 
onal nos anos recentes ${ }^{16,23,24}$, o que representa não apenas a crescente visibilidade da questão alimentar e nutricional da população brasileira, como também a ideia da possi bilidade de obtenção de recursos para a viabilização de iniciativas que se propõem a responder aos problemas a ela relacionados. Para os meios científicos, significa a possibilidade de dar andamento mais sólido a estudos vinculados a esse tema.

Nada mais coerente, neste cenário, que um interesse crescente da comunidade científica pela temática SAN: os resultados mostram que, até 1989, foram criados seis desses grupos; durante os anos noventa, mais dezessete grupos; e, a partir do ano 2000, mais 49 grupos surgiram, de acordo com a base corrente em outubro de 2005.

\section{Dinamismo e consolidação da pesquisa}

Guimarães et al. ${ }^{9}$ afirmam que os doutorandos de hoje irão constituir os pesquisadores de amanhã de determinada área eque o dinamismo deuma pesquisa, ou seja, a intensidade de reprodução de sua força de trabal ho, pode ser expresso pela relação entre o número de doutorandose pesquisadores doutores. Além disso, consideram que a consolidação científica de uma área pode ser determinada a partir da relação entre o número de doutores e estudantes em todos os graus de qualificação. Grupos de pesquisa dinâmicose consolidados seriam aqueles formados por pesquisadores doutores, entre os quais seus líderes, com alunos de iniciação científica, mestrandos, doutorandos e técnicos; estariam, portanto, associados, de alguma forma sólida, a cursos de graduação e a programas de pós-graduação, formando regularmentee com agilidade novos pesquisadores aptos a conduzir, de per si, trabalhos de geração e disseminação detecnologias, conhecimentos e saberes.

Em seu estudo acerca da epidemiologia, Guimarães et al. ${ }^{9}$ concluíram que essa pesquisa no Brasil apresenta alto dinamismo, porém ainda está longe de possuir a consolidação de certas áreas. Prado e Sayd ${ }^{10,11}$, estudando a pesquisa nacional sobre envelhecimento, verificaram um baixo dinamismo e a necessidade de um percurso ainda maior do que para a epidemiologia, até a consolidação científica.

A Tabela 3 traz resultados que indicam fragilidades importantes na pesquisa relativa à SAN. Apesar do crescimento recente do número de grupos, estes são, em média, pouco consolidados e apresentam baixo dinamismo. Observe-se que, em 2004, mais de 75\% desses grupos não contavam com doutorandos em seus quadros.

Se o dinamismo ea consolidação da pesquisa passam, necessariamente, pela articulação com programas de pós-graduação, cabe mencionar alguns dados sobre os programas de formação de mestres e doutores em Nutrição no Brasil, enfatizando queesteconjunto correspondea apenas uma parcela do universo da pós-graduação queenvolveSAN.

Tabela 3. Relações entre estudantes e pesquisadores nos grupos de pesquisa em grandes áreas do conhecimento no total do diretório ${ }^{12}$, em epidemiologia ${ }^{9}$, em gerontologia ${ }^{10}$ e nos grupos que mencionam segurança alimentar e nutricional ${ }^{8}, 2000$ a 2004, Brasil.

\begin{tabular}{|c|c|c|c|c|c|c|}
\hline \multirow{3}{*}{ Grande área } & \multicolumn{6}{|c|}{ Anos do censo } \\
\hline & 2000 & 2002 & 2004 & 2000 & 2002 & 2004 \\
\hline & \multicolumn{3}{|c|}{ Dinamismo $^{\mathrm{a}}$} & \multicolumn{3}{|c|}{ Consolidação $^{b}$} \\
\hline Ciências humanas & 0,36 & 0,32 & 0,31 & 0,17 & 0,18 & 0,15 \\
\hline Ciências biológicas & 0,52 & 0,51 & 0,41 & 0,22 & 0,26 & 0,19 \\
\hline Engenharias e ciências da computação & 0,58 & 0,50 & 0,39 & 0,25 & 0,29 & 0,19 \\
\hline Ciências da saúde & 0,34 & 0,28 & 0,22 & 0,19 & 0,18 & 0,12 \\
\hline Ciências exatas e da terra & 0,49 & 0,43 & 0,32 & 0,30 & 0,32 & 0,21 \\
\hline Ciências agrárias & 0,36 & 0,35 & 0,28 & 0,23 & 0,26 & 0,17 \\
\hline Ciências sociais e aplicadas & 0,19 & 0,16 & 0,16 & 0,10 & 0,11 & 0,09 \\
\hline Linguística, Letras e Artes & 0,33 & 0,35 & 0,30 & 0,18 & 0,21 & 0,15 \\
\hline Epidemiologia & 0,40 & - & - & 0,18 & - & - \\
\hline Gerontologia & 0,26 & - & - & 0,14 & - & - \\
\hline SAN & 0,80 & 0,45 & 0,10 & 0,17 & 0,36 & 0,14 \\
\hline Todas as áreas & 0,43 & 0,38 & 0,31 & 0,22 & 0,24 & 0,16 \\
\hline
\end{tabular}

a. Dinamismo $=$ Doutorandos $/$ Pesquisadores doutores b. Consolidação $=$ D outorandos $/$ Total de estudantes 
Estudo envolvendo a pós-graduação brasileira em alimentos enutrição ${ }^{25}$ indicou a existência de 39 programas credenciados pela Coordenação de Aperfeiçoamento de Pessoal de Nível Superior (Capes) do triênio 2001-2003.

$\mathrm{Kac}$ et al. ${ }^{26}$ estudaram, especificamente, doze programas de pós-graduação no campo da N utrição: onze mestrados acadêmicos e um profissional e cinco cursos de doutorado. Os autores entendem que esses programas são recentes, que correspondem a um número reduzido diante do complexo e preocupante cenário al imentar enutricional do país e quea sua "evolução no sentido da excelência passa, necessariamente, por um substancial aumento na produção intelectual".

São considerações que vão ao encontro dos achados neste estudo sobre os grupos de pesquisa sobreSAN, indicando a necessidade dequeas agências defomento à pesquisa eos organismosresponsáveis pela formação no plano da pós-graduação façam investimentos em iniciativas que busquem responder às demandas nacionais nesse campo.

\section{Áreas predominantes}

Ao escolher uma área predominante e indicá-la nos formulários de coleta de dados do diretório, o líder define a posição de seu grupo na árvore do conhecimento.

No total da base de dados do diretório, a grandeárea predominante do conhecimento que integra o maior número de grupos de pesquisa éa das ciências da saúde, seguida das ciências humanas.

No presente levantamento, foram identificados grupos com linhas de pesquisa referentes à SAN em cinco das oito grandes áreas. Distintamente do conjunto nacional, aqui a grande área predominante, de 2000 a 2005, correspondeà das ciências agrárias, seguida das ciências da saúde, com uma grande distância, e, por fim, um terceiro e pequeno agrupamento situado no campo das humanidades. Do ano de 2000 ao de 2005, prepondera àárea de ciência e tecnologia dos alimentos, seguida da Nutrição (Tabela 4).

Tabela 4. Distribuição dos grupos de pesquisa no total do diretório por ano do censo ${ }^{13}$ e dos que mencionam segurança alimentar e nutricional por ano do censo e na base corrente ${ }^{3}$ segundo grande áreas e áreas predominantes do conhecimento, 2000 a 2005, Brasil.

\begin{tabular}{|c|c|c|c|c|c|c|c|}
\hline \multirow{3}{*}{$\begin{array}{c}\text { Grande área } \\
\text { Área predominante }\end{array}$} & \multicolumn{6}{|c|}{ Anos do censo } & \multirow{3}{*}{$\begin{array}{c}\text { Base } \\
\text { corrente } \\
\text { outubro de } \\
2005 \text { SAN }\end{array}$} \\
\hline & \multicolumn{2}{|c|}{2000} & \multicolumn{2}{|c|}{2002} & \multicolumn{2}{|c|}{2004} & \\
\hline & Diretório & SAN & Diretório & SAN & Diretório & SAN & \\
\hline Ciências da saúde & 1.832 & 1 & 2.513 & 2 & 3.371 & 16 & 22 \\
\hline Nutrição & 69 & 1 & 100 & 2 & 124 & 12 & 18 \\
\hline Saúde coletiva & 289 & 0 & 388 & 0 & 521 & 3 & 3 \\
\hline Farmácia & 103 & 0 & 171 & 0 & 245 & 1 & 1 \\
\hline Ciências humanas & 1.711 & 0 & 2.399 & 0 & 3.088 & 0 & 2 \\
\hline Geografia & 71 & 0 & 111 & 0 & 150 & 0 & 1 \\
\hline Sociologia & 187 & 0 & 240 & 0 & 296 & 0 & 1 \\
\hline Ciências exatas e da terra & 1.826 & 0 & 2.243 & 0 & 2.826 & 0 & 0 \\
\hline Ciências biológicas & 1.720 & 0 & 2.126 & 1 & 2.561 & 2 & 3 \\
\hline Microbiologia & 179 & 0 & 220 & 1 & 271 & 2 & 3 \\
\hline Engenharias e ciências da computação & 1.812 & 0 & 2.051 & 0 & 2.454 & 0 & 0 \\
\hline Ciências sociais aplicadas & 930 & 0 & 1.429 & 1 & 2.120 & 2 & 5 \\
\hline Economia & 221 & 0 & 272 & 1 & 326 & 2 & 4 \\
\hline Direito & 103 & 0 & 206 & 0 & 366 & 0 & 1 \\
\hline Ciências agrárias & 1.352 & 2 & 1.653 & 8 & 1.997 & 21 & 40 \\
\hline Ciência e tecnologia dos alimentos & 193 & 2 & 241 & 6 & 297 & 16 & 29 \\
\hline M edicina veterinária & 238 & 0 & 280 & 1 & 340 & 1 & 4 \\
\hline Zootecnia & 182 & 0 & 216 & 0 & 261 & 1 & 3 \\
\hline Agronomia & 535 & 0 & 666 & 1 & 793 & 3 & 3 \\
\hline Recursos pesqueiros e Engenharia de pesca & 58 & 0 & 52 & 0 & 73 & 0 & 1 \\
\hline Linguística, Letras e Artes & 577 & 0 & 744 & 0 & 1.053 & 0 & 0 \\
\hline Total das grandes áreas & 11.760 & 3 & 15.158 & 12 & 19.470 & 41 & 72 \\
\hline
\end{tabular}

a. Estão apresentadas apenas as áreas para as quais houvemenção a SAN . 
SAN, em seu conceito amplo e multifacético, engloba: (a) um componente alimentar, que se refere à produção, disponibilidade, comercialização, acesso e consumo do alimento saudável e (b) um componente nutricional relacionado às práticas alimentares e à utilização biológica dos alimentos (indicadores antropométricos, bioquímicos, etc.) contemplando todas as fases do ciclo de vida e estendendo-se a grupos institucionalizados (creches, escolas, asilos, etc.) ${ }^{5}$.

A leitura atenta das denominações utilizadas para apresentar os grupos e linhas de pesquisa identificados no presente estudo para a área de ciência etecnologia dos alimentos pareceindicar uma certa tônica importante sobreiniciativas que valorizam a qualidade sanitária dos alimentos, visando ao mercado interno einternacional, entendidas como uma certa vocação econômica nacional.

É oportuno registrar as observações de Guimarãe ${ }^{27}$ acerca do privilégio que a política de ciência e tecnologia confere, no Brasil, aos setores de atividade econômica como base de sua concepção e orientação. 0 autor considera que, embora compreensível como direção geral, esse caminho tem deixado delado outros setores fundamentais que deveriam ocupar lugar mais central na pesquisa: a atividade social, particularmente, a alimentação, a saúde, a habitação e a educação: Esse olhar da política de C\&T em dire ção às políticas sociais possui, também, a virtude deoperar um deslocamento na di reção de um novo projeto nacional no qual possa ser mais valorizado o mercado interno e as necessidades da população brasileira. Por exemplo, no que se refereá alimentação, ao lado de aumentar a competitividade das commodities brasileiras de exportação (o que é, sem dúvida, importante), será essencial inaugurar linhas deapoio à pesquisa com vistas, por exemplo, a uma política de segurança alimentar.

No sentido da atenção à saúde das populações, encontra-se uma outra parte da pesquisa relativa à SAN: aquela que se volta especificamente para a análise e/ou monitoramento do estado nutricional de populações, discussão de políticas e programas de alimentação e nutrição e promoção da alimentação saudável. Correspondeao componentenutricional daSAN - aquele relacionado com a nutrição, ou seja, a utilização biológica dos alimentos (indicadores antropométricos, bioquímicos, etc.) - e que se situa bem mais nos grupos identificados nas áreas da Nutrição e da Saúde Coletiva.

Vale registrar a perspectiva deSaúde Coletiva apresentada por $\mathrm{M}$ inayo $^{28}$, que muito bem se presta a fundamentar a pesquisa relativa ao componente nutricional da SAN, em seu horizonte de valorização de políticas sociais e que reclamam a ética e a realização do ser humano como pilares de sua implementação: A visão do campo da SaúdeColetiva équeo progresso econômico deve estar a serviço da equidade, ea ciência etecnologia relacionadas a um projeto de desenvolvimento ancorado no caráter dinâmico dos processos avaliativos de prioridades. $\mathrm{O}$ u seja, sob qualquer aspecto que se analise, essa área de conhecimentos e de práticas não se coloca à margem do desenvolvimento da biologia, da genética, das ciências ambientais, da medicina. Porém vincula esses avanços a seu sentido social, político e de direito universal.

Finalmente, cabe registrar a existência um pequeno número de grupos situados no campo das humanidades que abordam temáticas absolutamente afins ao conceito de SAN que tomamos por referência. Esses grupos propõem o desenvolvimento de estudos que se articulam com o componente alimentar da SAN ou com o que poderíamos denominar alimentação entendida como espaço das relações econômicas, culturais e sociais construídas ao longo da história entre 0 homem e 0 alimento.

Em suma, os aspectos alimentares da SAN parecem encontrar-semais presentes entreos grupos situados nas grandes áreas das ciências agrárias e das humanidades. Os grupos que se situam na área da ciência e tecnologia dos alimentos encontram-se mais voltados para a parcela alimento, numa visão de SAN mais restrita e focada em sua produção para a comercialização. Os grupos inseridos nas humanidades voltam-se mais para a formulação eimplementação depolíticas, ações, intervenções de SAN nos planos nacional, regional e local, demonstrando identidade com uma perspectiva mais ampliada do componente alimentar da SAN ou para a alimentação.

Os aspectos nutricionais da SAN - ou os estudos sobre a nutrição -, parecem ser tratados, predominantemente, entre os grupos que se situam na grande área da Saúde, em particular nas áreas de N utrição e Saúde Coletiva.

\section{Produção científica}

Considerando a produção científica acumulada desde a criação dos grupos em tela até 0 censo de 2004, observa-seque apenas quinzegrupos $(37 \%)$ contavam com alguma publicação (artigo nacional ou internacional, livro ou capítulo de livro já publicado) ou orientação de trabalho de pós-graduação stricto senso concluído 
sobre esse tema; destes, quatro grupos juntos deram origem a 63 trabal hos, o que corresponde à cerca de $68 \%$ dos 93 considerados para esta discussão. Esse seria, talvez, o núcleo principal, a massa crítica mais envolvida com a geração e condução de debates sobre SAN no Brasil até meados de 2003, quando foi fechada a coleta de dados para o censo de 2004.

Essa característica permite pensar, a partir de Bourdieu ${ }^{29}$, em um campo mais central da produção nacional de saberes e conhecimentos voltados para SAN, que corresponderia aos grupos de pesquisa com produção científica mais expressiva, ena existência concomitante, no seu entorno, de campos de interação mais duradouros ou fugazes, numa dinâmica queexigeacompanhamento ediscussão permanentes de suas alterações einterações, consensos, conflitos e contradições.

Considerando que é crescente o número de grupos de pesquisa que mencionam $\mathrm{SAN}^{30}$ e que estes foram criados nos anos bem recentes, parece razoável pensar que ainda não tenha havido tempo hábil para a publicação de livros ou artigos ou para a geração de outros produtos e que o futuro próximo pode revelar uma produção científica também crescente.

Os grupos mais produtivos encontram-se ligados a programas de pós-graduação stricto sensu, o queestá diretamente vinculado à geração de dissertações e teses e caracterizam-se por apresentar melhores indicadores de dinamismo e de consolidação científica.

O levantamento da produção científica originária desses grupos possibilitou o desenho de três principais agrupamentos temáticos.

O primeiro agrupamento temático - com 31 produtos - refere-se, principalmente, à segurança do alimento, sua qualidade sanitária e aspectos relativos aos processos tecnológicos de sua produção; são os temas de interesse que mantém correspondência com a área de ciência etecnologia dos alimentos, área predominante entre os grupos de pesquisa que mencionam SAN voltada para o domínio dos estudos sobre o alimento. Alguns exemplos de expressões presentes em títulos desses produtos são bastante ilustrativos: "Qualidade de leite pasteurizado eUAT comercializados [...] quanto a alguns indicadores de se gurança alimentar"; "Biocontrole de doenças pós-colheita de frutas por leveduras: perspectivas de aplicação e segurança alimentar"; "Condições de higiene de cachorros-quentes comercializados em vias públicas"; "Envase de palmito de pupunha em vidro"; O cratoxina A em café"; “Re síduos de agrotóxicos em alimentos; segurança alimentar e 0 emprego de aditivos em alimentos", etc.

Um segundo agrupamento temático - com 28 produtos - diz respeito a orientações relativas à alimentação saudável voltadas para grupos da população (aleitamento materno, crianças, escolares, adolescentes, adultos, idosos, indígenas, quilombolas, etc.); inclui estudos sobre estado nutricional de populações e sobre a implantação e a implementação do Sistema de Vigilância Alimentar e Nutricional (SISVAN). Há aqui correspondência com a área da Nutrição, em particular, aquela que faz interface com a Saúde Coletiva e evidenciam-se proximidades com o domínio da nutrição, ou seja, com o espaço das relações de aproveitamento denutrientes pelo corpo normal ou patológico e de suas consequências para o estado nutricional de indivíduos ou populações, bem como das orientações nutricionais para que patamares mais adequados de saúde sejam alcançados. Os títulos desses trabalhos são bem característicos: "Aleitamento materno e a prática de desmame em [ ...] comunidades rurais"; "Nutrição e estilos saudáveis de vida"; "Alimentação saudável da gestante"; ; "Alimentação saudável do pré-escolar, escolar e adolescente"; "Alimentação saudável na terceira idade"; "Segurança alimentar e nutricional na atenção básica em saúde"; "Os desafios do SI SVAN na segurança alimentar e nutricional no Brasil"; "Desnutrição materno infantil na década de 90 ", entre outros similares.

Um terceiro e último agrupamento temático - com 34 produtos - trata de questões mais abrangentes como o direito humano à al imentação saudável, macropolíticas associadas à implantação da SAN no Brasil, desenvolvimento econômico em suas relações com as desigualdades sociais, a fome e a pobreza ou questões relativas à organização de produção, distribuição e acesso a alimentos em regiões ou municípios do país. Temas bem afeitos ao campo das humanidades e ao domínio da alimentação. Os extratos de títulos dos trabalhos oriundos desses grupos são bem ilustrativos: "N ovo contexto internacional do abastecimento eda segurança alimentar; efeitos distributivos da autocapacidade alimentar no Brasil"; "Programa Fome Zero no contexto das políticas sociais no Brasil"; "Políticas municipais er regionais de abastecimento esegurança alimentar; direitos humanos à segurança alimentar e nutricional no Brasil"; "Economia de rede, o papel da distribuição e a problemática da segurança alimentar"; "Diversidad, desigualdades y la cuestion alimentaria"; "Food safety issues in developing nations: a case study of Brazil". 
Uma referência importante para a discussão desses agrupamentos temáticos corresponde ao documento intitulado "Princípios e D iretrizes de uma Política de Segurança Alimentar e Nutricional" 4 , produzido por solicitação do CONSEA com vistas a subsidiar debates e, ao mesmo tempo, indicar elementos principais para a formulação da estratégia que daria suporteà implementação da SAN no Brasil. Ele oferece um certo contorno temático que permite o estabelecimento de correlação com os campos do conhecimento e sua respectiva produção científica aqui em questão. Desse documento do CONSEA, é possível visualizar dois grandes campos de reflexão e ação.

Um primeiro campo de natureza bem ampla equeremete a componentes macropolíticos, entendidos como fundamentos para a implantação da SAN - cujos temas estão fortementefocados no domínio da alimentação e abordam forma apenas tangente 0 alimento:

A construção de uma política de Segurança Alimentar. 0 direito humano à alimentação. Sistema agroalimentar mundial, organismos e acordos internacionais. Componentes de Segurança Alimentar e Nutricional em um Plano Nacional de Reforma A grária. Políticas de apoio e fortalecimento da agricultura familiar e do agro extrativismo. Recursos genéticos, sementes e a questão dos transgênicos. A cesso e uso dos recursos naturais e da água. Abastecimento alimentar eagricultura urbana. Programas de suplementação de renda e de suplementaç̧ão alimentar. M utirões, col eta e doação de alimentos e ações em situações emergenciais. Participação social nas políticas de segurança alimentar e nutricional. Institucionalidade das políticas de segurança alimentar e nutricional.

Considerando as preocupações aí presentes com a produção, disponibilidade, comercialização, acesso e consumo do alimento saudável, poder-se-ia traçar vínculos temáticos entre esse primeiro campo temático e o componente alimentar da SAN anteriormente mencionado.

E um segundo campo temático, corresponde a concepções e ações mais próximas ao componente nutricional - ou o domínio da nutrição -, está assim apresentado: Ações de promoção e vigilância em saúde e nutrição. Alimentação e educação em escolas e creches. Cultura alimentar. Alimentação emodos de vida saudáveis. Indicadores de segurança alimentar enutricional. A construção de instrumentos para avaliar e monitorar a situação deSAN, ações e os programas implantados.

As correlações entre esses dois grupos de temas colocados pelo CONSEA e os que foram identificados nos grupos de pesquisa que se pro- põem ao estudo da SAN parecem manifestar-se nos domínios da nutrição e da alimentação, indicando que há respostas sendo construídas pelos pesquisadores diante dessa demandas, mesmo que em caráter inicial.

Chama atenção o isolamento dos grupos de pesquisa mais situados no domínio do alimento em relação ao conceito de SAN; esses grupos, embora numericamente predominantes, geram trabalhos cujos objetos de estudo aparecem de forma bastante pontual na pauta indicada pelo CONSEA.

\section{Consideraçõesfinais}

0 conceito de SAN implica e reclama a integração intersetorial; nesse sentido, poder-se ia considerar animadora a existência de grupos de pesquisa que mencionam SAN em diversas áreas do conhecimento e que se encontram voltados para domínios que tratam dos alimentos, da alimentação e da nutrição.

Entretanto, percebe-se a predominância de grupos voltados para o domínio do alimento, que trabalham a partir do que nos parece um outro conceito de segurança alimentar mais voltado para a questão sanitária, para a inocuidade e para a qualidade do alimento com vistas à sua comercialização. U ma perspectiva muito importante, porém bastanteaquém das dimensões conceituais colocadas pela SAN .

Considera-se a necessidade de investimentos na integração dos componentes alimentar e nutricional da pesquisa sobre SAN, buscando reduzir o diferencial entre o foco econômico - da segurança e inocuidade do alimento - e o foco social - do direito humano à alimentação saudável, de forma a incrementar as buscas por respostas mais amplas para os problemas relativos acesso ao alimento, às carências nutricionais, à fome, valorizando as práticas saudáveis no campo da alimentação, respeitados seus constituintes culturais.

Quanto à organização interna desses grupos - presença de estudantes em diversos níveis de formação e de profissionais qualificados para os trabal hos científicos e tecnológicos - este estudo aponta insuficiências na formação de novos pesquisadores. Em outras palavras, a pesquisa sobre SAN no Brasil parece pouco dinâmica e demandando longo caminho para sua consolidação.

Assim, este trabal ho traz indicações de que a pesquisa sobre SAN não se encontra adequadamente dimensionada e qualificada em face da 
amplitude conceitual e da grandiosidade das questões relativas à SAN num país de dimensões continentais e mergulhado em desigualdades sociais tão profundas como o Brasil.

Ainda há muito a construir, pois os grupose linhas de pesquisa parecem ter sido constituídos na lógica da especificidade, bastante afastados da concepção de SAN como um campo de conhecimento amplo eintersetorial.

\section{Colaboradores}

SD Prado, AS Gugelmin, JK da Silva ePSG Olivares participaram da concepção e desenho do estudo, coleta e organização dos dados, análise e interpretação dos resultados e aprovação da versão final. RA de Mattos participou da análise e interpretação dos resultados e aprovação da versão final.

\section{Referências}

1. Prado SD. A pesquisa sobre Alimentos, Alimentação e Nutrição no Brasil: reflexões sobre a produção de conhecimento e saberes [projeto de pesquisa]. Rio de Janeiro: Instituto de Nutrição/U niversidade do Estado do Rio de Janeiro; 2005.

2. Valente FLS. Direito humano à alimentação: desafios e conquistas. São Paulo: Cortez; 2002.

3. Maluf RS, Menezes F, Valente FL. Contribuição ao tema da segurança alimentar no Brasil. Revista Cadernos de Debate 1996; 4:66-88.

4. Conselho Nacional de Segurança Alimentar e Nutricional. Princípios e Diretrizes de uma Política de Segurança Alimentar e N utricional. [acessado 2006 jun 05]. Disponível em: https://www.planalto.gov.br/ consea/static/documentos/LivroConsea. DocumentoReferencia.pdf

5. Conselho Nacional de Segurança Alimentar e Nutricional. Relatório Final da II Conferência Nacional de Segurança Alimentar e N utricional. [acessado 2006 jun 05]. Disponível em: https://www. planalto.gov.br/ consea/static/documentos/IIConferencia.pdf

6. Conselho Nacional de Segurança Alimentar e Nutricional. II Conferência + 2. Encontro $\mathrm{Nacional}$ de Segurança Alimentar e Nutricional. Subsídios para os eventos preparatórios. [acessado 2006 jun 05]. Disponível em: https://www.planalto.gov.br/ consea/static/documentos/D ocumento_base.pdf

7. Brasil. Lei $n^{\circ}$ 11.346. Cria o Sistema Nacional de Segurança Alimentar e Nutricional (SISAN) com vistas em assegurar o direito humano à alimentação e dá outras providências. Diário Oficial da União 2006; 15 set.

8. Conselho Nacional de Desenvolvimento Científico e Tecnológico. Diretório dos Grupos de Pesquisa. [acessado 2006 mar 30]. Disponível em: http:// lattes.cnpq.br/grupos/grup_apresent.htm

9. Guimarães R, Lourenço R, Cosac S. A pesquisa em epidemiologia no Brasil. Rev. Saude Publica 2001; 35(4):321-340.

10. Prado SD, Sayd JD. A pesquisa sobre envelhecimento humano no Brasil: grupos e linhas de pesquisa. Cien Saude Colet 2004; 9(1):57-67.

11. Prado SD, Sayd JD. A pesquisa sobre envelhecimento humano no Brasil: pesquisadores, temas e tendências. Cien Saude Colet 2004; 9(3):763-772. 
12. Conselho Nacional de Desenvolvimento Científico e Tecnológico (CNPq). Diretório dos Grupos de Pesquisa Séries básicas. Principais dimensões. [acessado 2006 mar 30]. Disponível em: http:// lattes.cnpq.br/grupos/grup_apresent.htm

13. Marchelli PS. Formação de doutores no Brasil e no mundo: algumas comparações. Rev. Brasileira de Pós-Graduação 2005; 2(3):9-10.

14. Izique C. Produção crescente. Pesquisa FAPESP 2002; 81:18-22.

15. Castro J. Geografia da fome. Rio de Janeiro: Civilização Brasileira; 2002.

16. Vasconcelos FAG. Combate à fome no Brasil: uma análise histórica de Vargas a Lula. Rev. Nutr. 2005; 18(4):439-457.

17. Fundação Instituto Brasileiro de Geografia e Estatística. Estudo Nacional de Despesas Familiares. Rio de Janeiro: IBGE; 1978.

18. Instituto Nacional de Alimentação e Nutrição. Pesquisa Nacional de Saúde e Nutrição. Brasília: Instituto Nacional de Alimentação e Nutrição; 1989.

19. Fundação Instituto Brasileiro de Geografia e Estatística. Pesquisa de Orçamentos familiares. Rio de Janeiro: IBGE; 1986,1997.

20. Peliano AMTM 0 mapa da fome: subsídios à formulação de uma política de segurança alimentar. Brasília: IPEA; 1993.

21. Victora CG, Barros FC, Vaughan JP. Epidemiologia da desigualdade. São Paulo: Hucitec; 1988.

22. M onteiro CA. Saúde e nutrição das crianças de São Paulo. São Paulo: Hucitec/Edusp, 1988.

23. Yasbek MC. O programa fome zero no contexto das políticas sociais brasileiras. São Paulo Perspec. 2004; 18(2):104-112.

24. Galeazzi MA. Segurança alimentar e cidadania. São Paulo: Mercado das Letras; 1996.

25. Jordão AA, Garcia RWD, Marchini JS. Fator de impacto e pós-graduação stricto sensu em alimentos, nutrição e ciência e tecnologia de alimentos. Rev. Nutr. 2006; 19(6):793-802.

26. Kac G, Fialho E, Santos SM C. Panorama atual dos programas de pós-graduação em Nutrição no Brasil. Rev. Nutr. 2006; 19(6):771-784.

27. Guimarães R. Pesquisa em saúde e reforma sanitária. Ciência e Cultura 2005; 57(1):37-38.

28. M inayo MCS. Pós-Graduação em Saúde Coletiva: um projeto em construção. Cien Saude Colet 1997; 2(1/2):53-71.

29. Bourdieu P. O campo científico. In: Ortiz R, organizador. Pierre Bourdieu: sociologia. São Paulo: Ática; 1983.

30. Base SAN - Grupos de Pesquisa sobre Segurança Alimentar e Nutricional no Brasil. [acessado 2006 jun 05]. Disponível em: http://www.nutricao.uerj.br 